\title{
Review Paper on Real-Time Vehicle Classification and Counting via Low-Cost Collaborative Sensing
}

\author{
Prof. S. O. Dahad ${ }^{1}$, Ms. Shital Tayade ${ }^{2}$ \\ Assistant Professor, ENTC, GCOEJ, Jalgaon, India ${ }^{1}$ \\ M.Tech, ENTC, GCOEJ, Jalgaon, India ${ }^{2}$
}

\begin{abstract}
In the field of traffic-information acquisition, one pervasive solution is to use wireless sensor networks (WSNs) to realize vehicle classification and counting. By adopting heterogeneous sensors in a WSN, the potential of using complementary physical information to perform more complicated sensing computation can be explored. However, the collaboration among heterogeneous sensors, such as the collaborative sensing mechanism (CSM), is not well studied in current state-of-the-art research. In this paper, the authors design and implement EasiSee, a real-time vehicle classification and counting system based on WSNs. Contributions are as follows. First, the authors propose a CSM, which coordinates the power-hungry camera sensor and the power-efficient magnetic sensors, reducing the overall system energy consumption and maximizing system lifetime. Second, a robust vehicle image-processing algorithm, i.e., a low-cost image processing algorithm (LIPA), is proposed. LIPA reduces environment noise and interference with low computation complexity. In the verification section, the vehicle detection accuracy turned out to be $95.31 \%$, which pave the way for CSM. The time of image processing is around $200 \mathrm{ms,} \mathrm{which} \mathrm{indicates} \mathrm{that} \mathrm{the}$ LIPA is computationally economical. With the overall energy consumption reduced, EasiSee achieves classification accuracy of $93 \%$. Based on these experiments and analysis, the authors conclude that EasiSee is a practical and lowcost affordable solution for traffic-information acquisition. Vehicle identification or classification is one of the application areas that come under real time image processing. Vehicle recognition is having the significance in various applications including the traffic monitoring, load monitoring, number plate recognition, vehicle theft prevention, traffic violation detection, management of traffic etc. As the images are captured as primary data source, it can have number of associated impurities which include the background inclusion, object overlapping etc. Because of this, object detection and recognition is always a challenge in real time scenario. In present work, a robust feature based model is presented for feature extraction and classification of vehicle images. The presented model is applied on real time captured image to categorize the vehicle in light, medium and heavy vehicle. Firstly, the vehicle area segmentation is performed and later on the Gaussian filter is applied to extract the image features. This featured dataset is processed under Support Vector Machine (SVM) based distance analysis model for vehicle recognition and vehicle class identification.
\end{abstract}

Keywords: Support Vector Machine (SVM), low-cost image processing algorithm (LIPA), CSM, WSN.

\section{INTRODUCTION}

Vehicle recognition is having the significance in various basic process model for vehicle detection and applications including the traffic monitoring, load classification is as shown in Fig.1

monitoring, number plate recognition, vehicle theft Firstly, the image is captured from the real environment prevention, traffic violation detection, management of using high resolution cameras in real environment. As the traffic etc. Some of the parking areas also use the vehicle image is captured, it can have number of impurities or the recognition approach as the surveillance method to avoid background overlapping, therefore there is requirement to the vehicle theft [6]. Earlier sensors, radar, loop detector filter and enhance the image. Moreover to improve the were used for vehicle recognition approach but there image, the image transformation is applied which includes installation and maintenance cost were high so to the size level, contrast level, color level adjustments. Now overcome these drawbacks the authors use computer this filtered enhanced image is used as the main featured vision approach. These applications generally work in image for classification process. In next stage, the video form, as the video is captured in real time and background is separated from the raw input image. This transformed to the sequence of images.

These images can be processed to perform the vehicle identification or vehicle class type identification. The process of background separation comes under segmentation process. The high level segmentation is applied over the image to identify the vehicle region of interest (ROI) over the image. The vehicle area 
identification is done using the thresholding method which is identified and the final work is to perform the feature extraction. There are number of related methods to explore the low level features over the image and the same features includes edge detection, feature point identification etc. As the image set is transformed to the feature set, the final stage is to apply the classification algorithm. There are number of classifiers to perform the recognition or classification of image. These classifiers includes Linear Discriminant Analysis (LDA) classifier [1], Bayesian network ], SVM, neural network, etc. The same classification model can be applied over the image to perform the recognition and classification of vehicle.

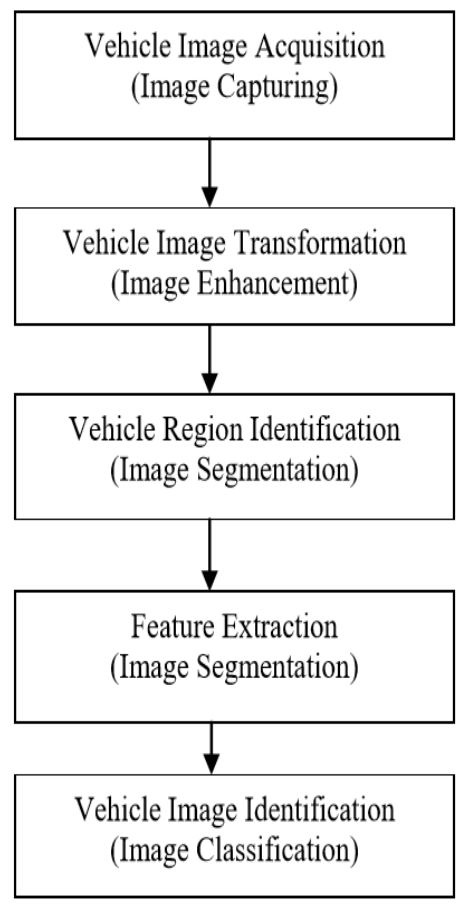

Fig.1 Vehicle Classification

\section{A METHODOLOGY AND COMPUTATIONAL MODEL OF PRESENT INVESTIGATION}

In present study, a feature adaptive intelligent model is presented for vehicle identification as well as vehicle class identification. Vehicle class is divided into light vehicle, medium vehicle and heavy vehicle. The study of research work is divided into three phases. In first phase the image is captured from real environment which may also suffers from number of associated challenges relative to the environment. These challenges include the background to the area, vehicle distance variation and the other vehicle movement [1820]. Because of this, there is the requirement to first identify the vehicle area or the object region based on which the actual classification process can be applied [18]. In preprocessing, improvement of image feature and separation of object part from background is done. To improve the image feature, image will be converted into grayscale and then histogram equalization is applied. As the features of image get improved, the next step is to locate vehicle over the image. To perform this, the thresholding based approach is applied along with morphological operator. The separation of background from foreground is done so that the actual object over the image will be identified. As the object or the vehicle is identified, the next work is to identify the vehicle features. In second phase, the feature extraction is performed over the image. To extract the features, the window adaptive model is applied in present investigation and feature extraction model is as shown in Fig. 2. In this stage, Gaussian filter and frequency analysis approach will be applied to extract the image features. This stage will transform the image set into statistical feature set. As the feature are extracted then the feature set are obtained for training and testing set and is obtained in the form of edge point the next stage is to perform vehicle classification.

In third phase, vehicle recognition and classification is done. To perform the classification SVM based weighted model and distance analysis approach is applied. As the feature set is obtained for training and testing dataset, the SVM classifier is applied to map the input image feature with dataset images. Based on this kernel mapped feature set, the weights over the images are applied and then compared with dataset images using distance based mapping. The maximum mapped input image over the dataset is considered as the recognized image. As the vehicle image is recognized, the next step is to identify the relative class and the training set mapped image class is considered as the class of input vehicle image. This SVM adaptive process is applied over the dataset till the complete recognition process is not completed.

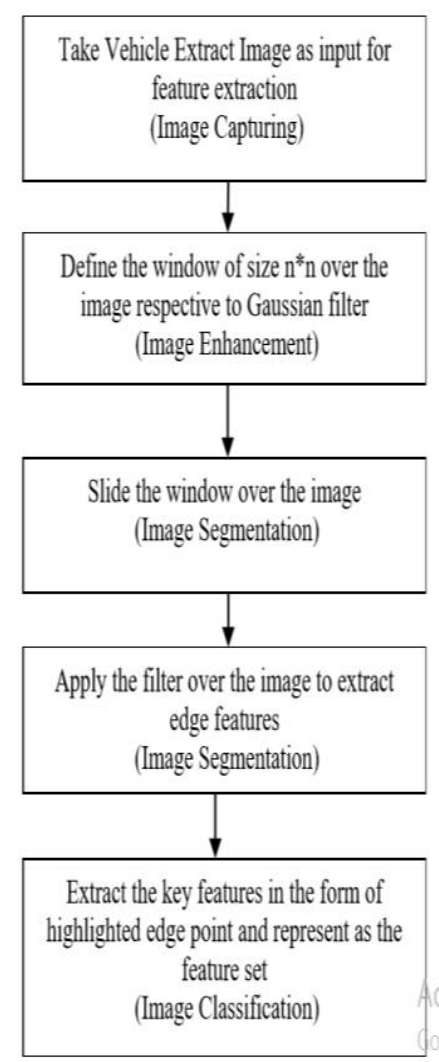

Fig. 2 Model for Extraction of Features of Image 
The work stages considered for present investigation are discussed as under:

(a) Get Vehicle Image The first requirement while working with real time application is to perform the effective capturing of image to acquire single vehicle in an image. The reliability of the recognition process depends on the image capturing. In this work, the real time image capturing is done using high resolution cameras. The image is captured under the background, distance and other vehicle analysis. (b) Improve Image Feature As the image is a raw image, it is required to improve and transform it to normalize image so as to improve features of image. This normalization process includes the conversion to grayscale form, resizing, and the enhancement of color and brightness for image. Beside this histogram equalization is applied to improve the feature of image. (c) Extraction of vehicle image To locate vehicle over image, thresholding based approach along with morphological operator is applied. The separation of background from foreground is done so that actual object over the image will be identified. As the object or vehicle is identified, the next work is to extract feature of image. (d) Feature Extraction After improving the image features, the window adaptive edge feature analysis model is applied. In this work, Gaussian filter is applied based on frequency analysis to acquire the features. The feature extraction is applied on training and testing set and feature set is obtained for both training and testing set. (e) Recognition After defining the training and testing set on vehicle images, the next step is to perform the recognition of vehicle. The vehicle recognition and classification is applied using SVM approach and it is a kernel based method. In first stage, the training set is trained over the SVM and after obtaining the network feature, the mapping of test image is done to training set via SVM. The distance adaptive mapping is here applied to perform the recognition of vehicle. As the maximum mapped vehicle is identified, the relative class such as light, medium and heavy vehicle is obtained. (f) SVM SVM is the classifier used in this work to identify the apnea and normal instances. This classifier is having the ability to provide the high accuracy in classification process while working with high dimensional data. This classifier also provides the modeling for diverse sources of data. SVM classifier actually comes under kernel based algorithmic approaches.

In this approach, the data dependency is also identified as the functional computation to generate the feature space. The advantages of the work are defined in terms of non linear decision boundaries defined under method specification for linear classification. This classifier is defined along with the specification of kernel function that itself provides the fixed dimensional vector representation with sequence generation and structural representation. Some of the most used Kernel functions are RBF Kernel, Polynomial Kernel, Fisher Kernel, String Kernel, and Sigmoid Kernel. This method effectively process on different decision functions to process on data values to identify the data criticality. The data usage based analysis can be obtained under environmental specification. The kernel specification is defined to control the classification process. There are different classification methods along with the specification of relative kernel parameters. The simplest form of SVM is called linear classifier that can be applied on balanced dataset. Further, the devised model based on Gaussian filter and SVM approaches is implemented in MATLAB environment. The next section of devised model presents a framework of results and discussion.

\section{LITERATURE SUMMARY}

The work model used LDA classifier for improving the classification rate. Probabilistic model to perform the vehicle prediction. The feature vector analysis with Bayesian network to perform the vehicle class identification. Vehicle detection and tracking under surveillance camera processing. Vehicle video analysis based on the frame averaging and provided the dimension specific analysis to extract the image features. Neural network for classification of vehicles and estimation of traffic on road. Surveillance of the videos and analyzed the key feature analysis for real video processing Classifies the vehicle depending on length using the algorithm developed. Comparing different classification approaches the HDBN provides better recognition rate. Model built with the help of Gaussian filter and SVM which provided better performance than model built with PCA and neural network. Utilized SVM and PCA classifiers for vehicle detection. video based vehicle tracking and classification applied on real time scenario and provided the Gaussian filter, Bayesian and fuzzy SVM for classification and recognition. Video stream processing using Bayesian network approach and defined the enhanced security mechanism to identify the target vehicle.

\section{SYSTEM DEVELOPMENT}

\section{A. Binary Classification}

Given training data (xi, yi) for $i=1 \ldots N$, with $x i \in R d$ and yi $\in\{-1,1\}$, learn a classifier $f(x)$ such that $f(x i)(\geq 0 y i=+1<0$ $y i=-1$ i.e. $y$ if $(x i)>0$ for a correct classification.

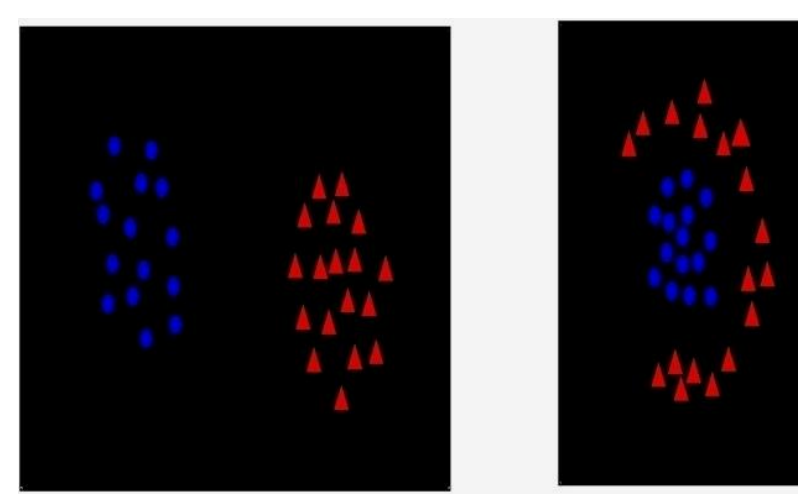

Fig 3 Binary Classification 


\section{Linear separability}

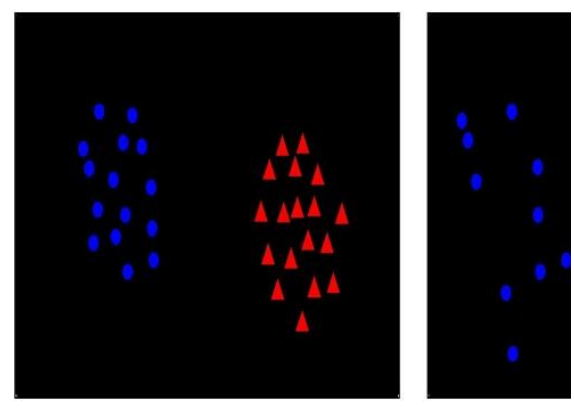

Fig. 4 Linearly Separable

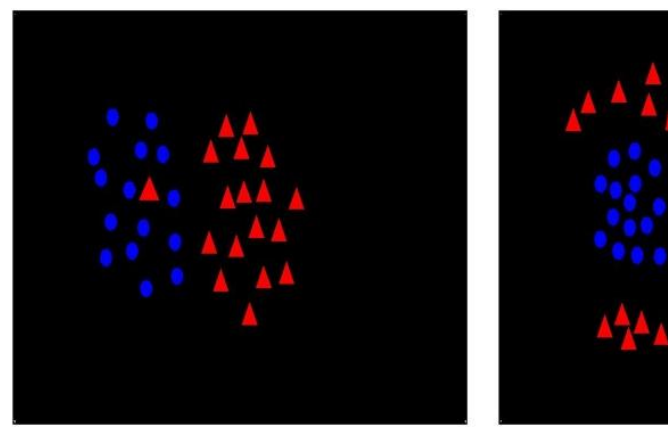

Fig. 5 Not Linearly Separable

\section{B. Linear Classifiers}

A linear classifier has the form $f(x)=w>x+b$

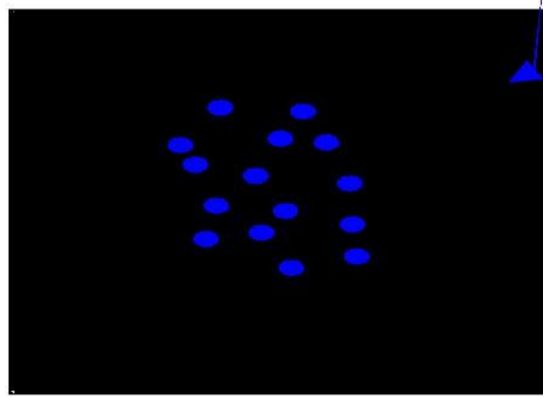

Fig. 6 Linear Classifiers(a)

- In $2 \mathrm{D}$ the discriminant is a line

- Is the normal to the line, and $\mathrm{b}$ the bias

- Is known as the weight vector

\section{Linear classifiers}

A linear classifier has the form $\mathrm{f}(\mathrm{x})=\mathrm{w}>\mathrm{x}+\mathrm{b}$

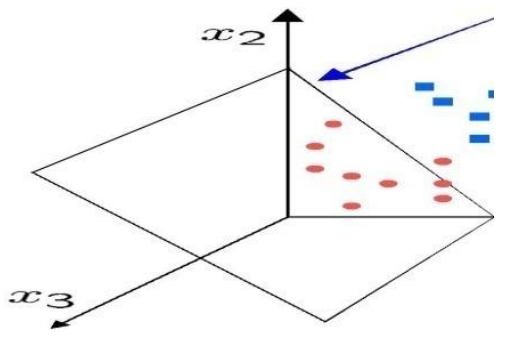

Fig. 7 Linear Classifiers(b)
-In $3 \mathrm{D}$ the discriminant is a plane, and in $\mathrm{nD}$ it is a hyperplane

For a K-NN classifier it was necessary to 'carry' the training data For a linear classifier, the training data is used to learn wand then discarded Only wis needed for classifying new data.

\section{C.The Perceptron Classifier}

Given linearly separable data xi labelled into two category find a weight vector $w$ such that the discriminant function $\mathrm{f}(\mathrm{xi})=\mathrm{w}>\mathrm{xi}+\mathrm{b} \quad$ separates the categories for $\mathrm{i}=1, \ldots, \mathrm{N}$ - How can we find this separating hyperplane ?

1) The Perceptron Algorithm:

Write classifier as $\mathrm{f}(\mathrm{xi})=\mathrm{w}^{\sim}>\tilde{x i}+\mathrm{w} 0=\mathrm{w}>\mathrm{xi}$ where $\mathrm{w}=(\mathrm{w}, \mathrm{w} 0), \mathrm{xi}=(\sim \mathrm{xi}, 1)$

- Initialize $\mathrm{w}=0$

- Cycle though the data points $\{\mathrm{xi}, \mathrm{yi}\}$

- If $x i$ is misclassified then $w \leftarrow w+\alpha \operatorname{sign}(\mathrm{f}(\mathrm{xi}$

- Until all the data is correctly classified

For example in $2 \mathrm{D}$

- Initialize $\mathrm{w}=0$

- Cycle though the data points $\{\mathrm{xi}$, yi $\}$

-Until all the data is correctly classified

-If $\mathrm{xi}$ is misclassified then $\mathrm{w} \leftarrow \mathrm{w}+\alpha \operatorname{sign}(\mathrm{f}(\mathrm{xi}$
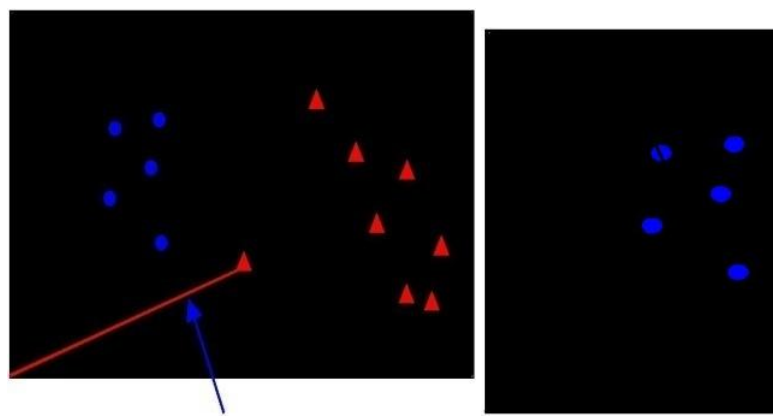

$\mathrm{NB}$ after convergence $\mathrm{w}=\mathrm{PNi}$ aixi

$$
\mathrm{w} \leftarrow \mathbf{w}
$$

Fig. 8 The Perceptron Algorithm

If the data is linearly separable, then the algorithm will c

- Convergence can be slow ...

- Separating line close to training data

- We would prefer a larger margin for generalization

- Maximum margin solution: most stable under perturbation

D. Target Classification

In this section, we introduce target classification using a boosting algorithm based method and image samples captured by camera sensors. Next, we first discuss feature extraction and feature selection, and then we introduce BbTC algorithm inspired by boosting algorithm. 
Perceptrons

Example

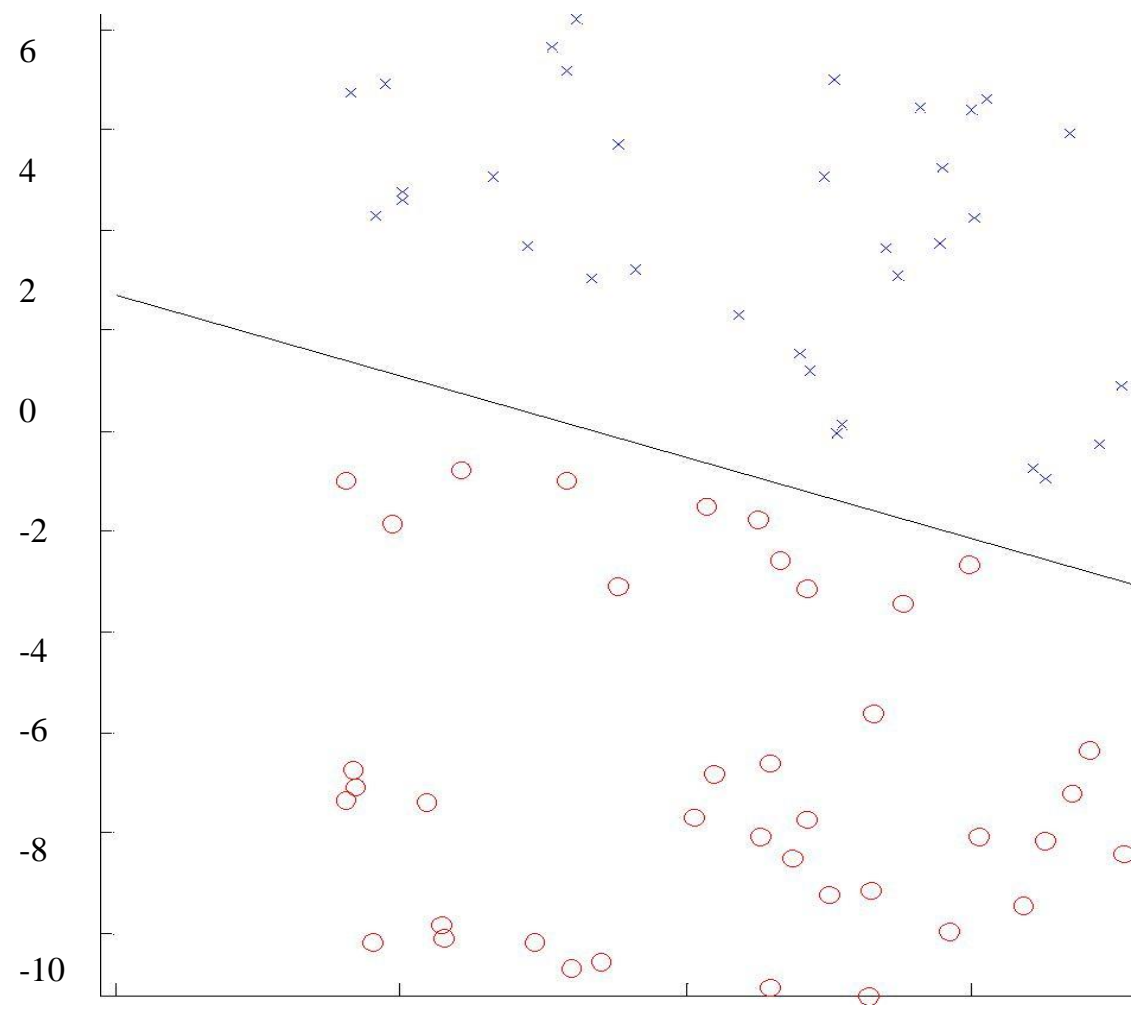

$-15$

$-10$

0

Fig. 9 The example of Perceptron

1) Feature Extraction and Selection Algorithm:

In order to make classification, we should extract feature from the integrated images captured by camera sensors through some basic image process methods. Note that we take bicycle and car as example for the target to illustrate our scheme in this paper.

\section{2) Feature Extraction:}

In this paper, to reduce the computation overhead, we first make mean compression for the original image. Then we make background subtraction, first-order gradient, and thresholds binarization for the compressed image respectively. The processed images of minibus, car and bicycle are shown. To extract the target feature, we obtain the vehicle outline as shown. The features including the length, width, area, perimeters, length/width (LHR), area/perimeter (PAR) of the vehicle are computed by counting the number of pixel in the vehicle outline according to features extraction algorithm

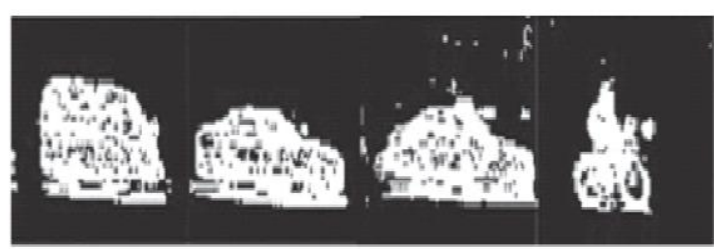

Fig.10 Image Processing

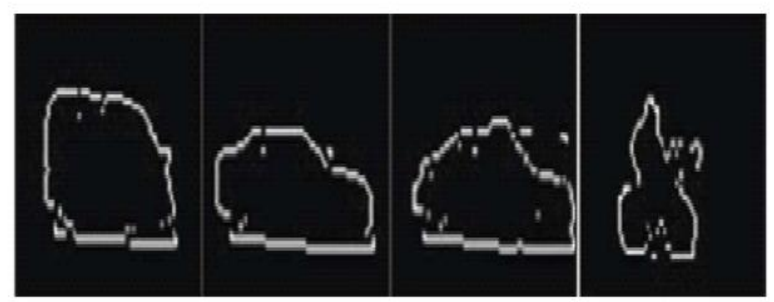

Fig.11 Vehicle Outline

\section{Extraction program}

clc;

clear all;

vidObj = VideoReader('viptraffic.avi');

$\%$ Read all video frames.

$\%$ Create an axes to display the video. Then, read video frames until the end of the file is reached.

$\mathrm{i}=1$;

currAxes = axes;

while hasFrame(vidObj)

vidFrame = readFrame $($ vidObj $)$;

filename $=[$ num2str(i) '.png'];

imwrite(vidFrame,filename,'png')

fprintf('writing frame \%d in to folder',i) ;

$\mathrm{i}=\mathrm{i}+1$;

end 
3) Feature Selection:

We manually collected sample images, including 259 bicycles, 140 cars. According to the analysis of features of the integrated images, we show that LHR and PAR are more helpful features in classification as shown. Therefo re, they are used to classify the vehicles.

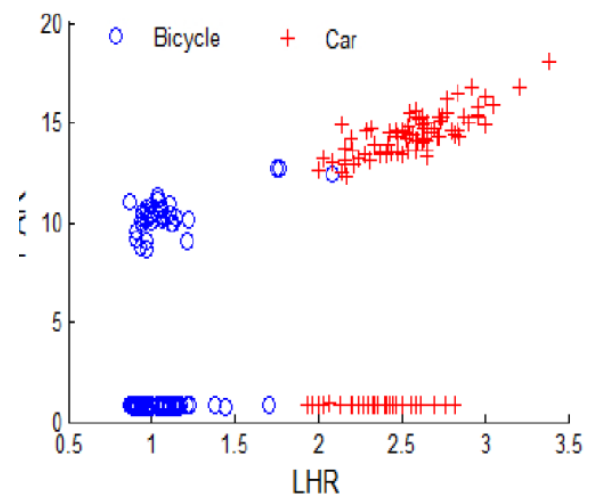

Fig. 12 Feature Selection

The work carried out in a real time image processing model is defined for vehicle recognition and vehicle class identification. The presented devised model is based on Gaussian filter \& SVM approach which is implemented in MATLAB environment and the same is applied on different vehicle datasets to obtain the analytical results. The presented work has defined hybrid model to perform the vehicle recognition and classification.

\section{REFERENCES}

[1] C. J. C. Burges. A tutorial on support vector machines for pattern recognition. Data Mining and Knowledge Discovery, 2(2):121-167, 1998.

[2] C.-C. Chang and C.-J. Lin. LIBSVM: a library for support vector machines, 2001. Software available at http://www.csie.ntu.edu.tw/ cjlin/libsvm.

[3] S. Gupte, O. Masoud, R. Martin, and N. Papanikolopoulos. Detection and classification of vehicles. IEEE Transactions on Intelligent Transportation Systems, 3(1):37-47, March 2002.

[4] S. S. Keerthi and C.-J. Lin. Asymptotic behaviors of support vector machines with gaussian kernel. Neural Computation, 15(7):1667$1689,2003$.

[5] M. Kirby and L. Sirovich. Application of the karhun enloeve procedure for the characterization of human faces. IEEE Transactions on Pattern Analysis and Machine Intelligence, 12(1):103-108, 1990

[6] K. Robert, N.Gheissari, and R.Hartley. Video Processing for Traffic Surveillance, 2006. Technical Report at the National ICT of Australia, http://www.nicta.com.au/.

[7] M. Turkand A. Pentland. Eigen faces for recognition. Journal of Cognitive Neuroscience, 3(1):71-86, 1991.

[8] S. Zehang, G. Bebis, and R. Miller. On-road vehicle detection using evolutionary gaborfilter optimization. IEEE Transactions on Intelligent Transportation Systems, 6(2):125-137, 2005.

[9] C. Zhang, X. Chen, and W. B. Chen. A pca-based vehicle classification framework. IEEE International Conference on Data Engineering, pages 17-17, 2006. 